\title{
Development of a Slender Continuum Robotic System for On-wing Inspection/Repair of Gas Turbine Engines
}

\author{
D. Axinte, X. Dong, D. Palmer, S. Cobos, M. Raffles, A. Rabani, J. Kell
}

\begin{abstract}
The maintenance works (e.g. inspection, repair) of aero-engines while still attached on the airframes requires a desirable approach since this can significantly shorten both the time and cost of such interventions as the aerospace industry commonly operates based on the generic concept "power by the hour". However, navigating and performing a multi-axis movement of an end-effector in a very constrained environment such as gas turbine engines is a challenging task. This paper reports on the development of a highly flexible slender (i.e. low diameter-to-length ratios) continuum robot of 25 degrees of freedom capable to uncoil from a drum to provide the feeding motion needed to navigate into crammed environments and then perform, with its last 6 DoF, complex trajectories with a camera equipped machining end-effector for allowing in-situ interventions at a low-pressure compressor of a gas turbine engine. This continuum robot is a compact system and presents a set of innovative mechatronics solutions such as: (i) twin commanding cables to minimise the number of actuators; (ii) twin compliant joints to enable large bending angles ( $\pm 90 \mathrm{deg})$ arranged on a tapered structure (start from $40 \mathrm{~mm}$ to $13 \mathrm{~mm}$ at its end); (iii) feeding motion provided by a rotating drum for coiling/uncoiling the continuum robot; (iv) machining end-effector equipped with vision system. To be able to achieve the in-situ maintenance tasks, a set of innovative control algorithms to enable the navigation and end-effector path generation have been developed and implemented. Finally, the continuum robot has been tested both for navigation and movement of the end-effector against a specified target within a gas turbine engine mock-up proving that: (i) max. deviations in navigation from the desired path $\left(1000 \mathrm{~mm}\right.$ length with bends between $45^{\circ}$ and $\left.90^{\circ}\right)$ are $\pm 10 \mathrm{~mm}$; (ii) max. errors in positioning the end-effector against a target situated at the end of navigation path is $1 \mathrm{~mm}$. Thus, this paper presents a compact continuum robot that could be considered as a step forward in providing aero-engine manufacturers with a solution to perform complex tasks in an invasive manner.
\end{abstract}

Index Terms - continuum robot; repair/maintenance of aero-engines; navigation; multi-axis manipulation of end-effectors

\section{INTRODUCTION}

$\mathrm{T}$ he in-situ repair of gas turbine engines while they are installed of the aircraft requires sets of specialised inspection/repair tools to be delivered to the desired positions without disassembly. Long term service agreements increase the importance of in-service inspection and subsequent repair activity. Early or unplanned removal causes significant customer disruption; this means that taking an engine off-wing not only results in significant disassembly/assembly costs but also in penalties for nonoperational time. Thus, in a highly competitive business such as aerospace power systems, the development of mechatronic/robotic systems able to navigate deep inside the geometrically intricate/crammed spaces of the engines and then, perform complex paths with end-effectors (e.g. cutting tools) could be of high technical advantage. Nevertheless, this is not an easily achievable task since it requires robots of "slender" designs, i.e. low diameter-to-length ratios, with many degrees of freedom to avoid collision with multiple obstacles (e.g. aerofoils), perform complex paths of end-effectors while being able to carry relevant payloads (e.g. tools) and position them accurately against target areas within the engines.

Despite of many reports in continuum robots that could be regarded as suitable systems to address these technical challenges, up to now, mostly borescopes and rigid-segmented boreblending tools are employed in most cases by the aero engine repair teams; commonly, through inspection holes positioned on the side of the engine casing, they can reach the target component and perform some corrective action. Some of boreblending tools [1] are constructed with small diameter (5 12mm) rigid links that generally present one Degree-of-Freedom (DoF). Others are flexible and have generally two DoFs [2,3], which allows the end of the tool bend in two directions. However, the current design of these invasive tooling are unable to cover a wide range of repair $\&$ maintenance works as they present limited DoFs and articulated lengths $(10 \sim 30 \mathrm{~mm})$, that prevent them to reach intervention places far away from the accessing ports while suffering from reduced accessibility in crammed spaces.

D. Axinte, X. Dong, D. Palmer, S. Cobos, M. Raffles, A. Rabani are with Rolls-Royce UTC in Manufacturing Technology University of Nottingham, Department of Mechanical, Materials and Manufacturing Engineering, Nottingham, NG7 2RD, United Kingdom (e-mail:Dragos.Axinte@nottingham.ac.uk).

J. Kell is with Repair Technology, Rolls-Royce plc, United Kingdom. 
On the other hand, over the years, the research work in the field of continuum robots have endeavoured to improve the capability of these systems in accessing constrained spaces by utilising increased number of degrees of freedom.

In this respect, some solutions using multiple rigid-joints per section for constructing continuum robots are available at various diameters $(9 \mathrm{~mm}$ to $60 \mathrm{~mm})$ and lengths $(200 \mathrm{~mm}$ to $1.5 \mathrm{~mm})$. These robots can access through small openings to enable inspecting jobs [4]. However, the rigid links could limit the accessibility within more intricate/crammed spaces [5] especially in those with tight succession of sharp bends.

Other approaches [6-9] use a series of super elastic NiTi alloys as backbone of continuum robots to enable enhanced articulation capabilities. Commonly, on these designs, one NiTi alloy rod is located at the centre of a succession of the disks that are actuated by cables to provide the bending of the continuum robots [10]. Furthermore, using this concept, it has been reported [6] that three NiTi rods can be used instead of actuating cables. Thus, enabling increased stiffness of the continuum robot. Although working continuum robotic solutions based on a central backbone have been reported, this design takes up the space in the centre of the manipulator, which prevents the passing through of utilities/end effectors, e.g. camera, illumination and machining spindle, which are of key importance to perform useful invasive operations. This is not to mention the tendency of such design to significantly twist around the central backbone when the continuum robot needs to take torques around the central axis [11]. To avoid this significant drawback, twin-joint NiTi alloy rods (positioned alternatively at 90deg in a succession of segments) have been used to design continuum robots [11]. Nevertheless, these continuum robots have been reported in short versions and without being equipped with feeding system, i.e. facility without which their autonomous utilisation for performing in-situ repairs/inspections within complex geometrical environments such as aeroengines is difficult.

Furthermore, other solutions utilise triple pneumatic actuators combined as articulated sections to build a flexible trunk [12] which can generate large longitudinal movement (ca. 45\% extension) and bending angle and thus, being used as handling assistant with appropriate $(0.5 \mathrm{~kg})$ payloads. Although this design presents high dexterity, with its large diameter of the bellows to ensure appropriate actuation forces, it renders as dimensionally unsuitable for repair/maintenance tasks within highly constrained environments such as aeroengines.

To address these needs and challenges, this paper reports on a design, mechatronic solutions and control methods of a slender (diameter/length of 0.023) continuum robot that provides adequate number (25) DoFs at small tip diameter (15mm) and high length $(1200 \mathrm{~mm})$, which make it able to perform inspection and machining tasks inside highly confined spaces, e.g. aeroengines. Thus, Section II of the paper details on the novelty of the design concept that consists in a succession of tapered segments of twin-compliant joint structures, which can significantly enhance the torsional stability while enabling the system to take appropriate payloads (max. $200 \mathrm{~g}$ ) at its tip. Section III details the static and kinematic models of the system, which have been utilised for the electro-mechanical design of continuum robot and the selection of the actuation system; additionally, it reports on the modelling of the compliant joint buckling was used to support the design criteria of the NiTi alloy twin compliant joint. Section IV presents in detail key aspects of the mechatronic design and realisation of the continuum arm, actuation pack, endeffector and architecture of the control system. Section V deals with the main modes of control that generates key commands to the continuum robot: tip-following (to enable the navigation); feeding-in/out (to enable advancement); machining (to enable path generation of the end-effector). In section VI, sets of targeted experiments to characterise the performance of the proposed continuum robot, including payload, navigation and inspection and machining tests are described. Finally, the conclusions are summarized in section VII.

\section{CONCEPT OF SLENDER CONTINUUM ROBOT FOR IN-SITU REPAIR OF AEROENGINES}

The design concept of the continuum robot has been driven by the technical requirements for performing in-situ (i.e. on-wing) repairs of aeroengines by accessing it from its front, i.e. fan section. Thus, to perform the required set of repair tasks, the slender of continuum robot was defined by the following limit specifications:

- min overall arm length $1200 \mathrm{~mm}$ - to ensure accessibility deep inside the crammed environment of the engine;

- max. tip diameter $15 \mathrm{~mm}$ (for at least $400 \mathrm{~mm}$ measured from its end) - to allow access in the crammed environment formed by the succession of static and rotating aerofoils of the engine;

min. bend angle per section \pm 90 deg. - to ensure that tight change in direction during the navigation into the engine;

- min. payload at the tip $0.250 \mathrm{~g}$ - to ensure appropriate end-effectors (e.g. machining spindle) can be carried;

- $\min .25 \mathrm{DoF}$ - to enable the feeding and the navigation of the continuum arm into intricate geometry environments and then to perform complex paths with the end-effectors for performing the repair tasks;

- max. dimension and weight of the actuation: pack $250 \mathrm{~mm}$ diameter (with $161 \mathrm{~mm}$ height) and $7.5 \mathrm{~kg}$ respectively - to allow the system being attached to other (mobile) robotic platforms (e.g. walking hexapods) and thus, enhancing the capabilities of the system.

To achieve these technical challenges, the slender continuum robot relies on a concept which key characteristics are discussed below and partially captured in schematic representation of Figure 1.

The preliminary simulation of the most challenging location into the aeroengine environment where the in-situ intervention 
could take place lead to the conclusion that the continuum robot would need to have 24 DoFs allocated to twelve-section, each of them actuated by steel cable. To ensure the high bending angles while enhancing torsional stability relative to the axis of the structure, a twin compliant joints (alternating at 90deg to account for 2 DoF each section) structure has been adopted (see Figure 1(a)). In order to make the deflections of the structure more uniform along its length, when the payload is applied at the tip, a tapered design has been adopted. For enhancing the stiffness of the structure at its base the dimensions and cross-section of the compliant joints have been altered per each section (e.g. base sections having lamellae joints while the tip sections rod joints see detail in Figure 1(b)); this concept also avoids the buckling of the structure at the base sections which accumulate higher compressive loads due to the need to support tensions of the actuating cables from "upstream" sections. This twin-compliant joint structure not only provides large bending angles (theoretically up to $\pm 90 \mathrm{deg} / \mathrm{section}$ ) but is also advantageous in allowing the provision of a working channel along the centre of the continuum arm which can be used for delivery of various endeffectors to the tip of the arm.

As the actuation pack needed to be compact, the large strokes of the actuating cables (attached to end-disks of each section) of the two pairs of cables system (see detail - Figure 1(c)) required a special design of a spooling system (detailed design in section IV) to allow winding/unwinding of equal cable lengths for a given rotation, that was used in conjunction for one motor. This paired cable design for a single spool also enables a constant tension applied on the cable (95\% of the cable tension force can be maintained in the work volume [11]). This is not to mention that compared with the concept of three actuators per section, this design reduces the number of actuators, rendering less weight of the actuating pack while simplifying the control of the continuum robot.

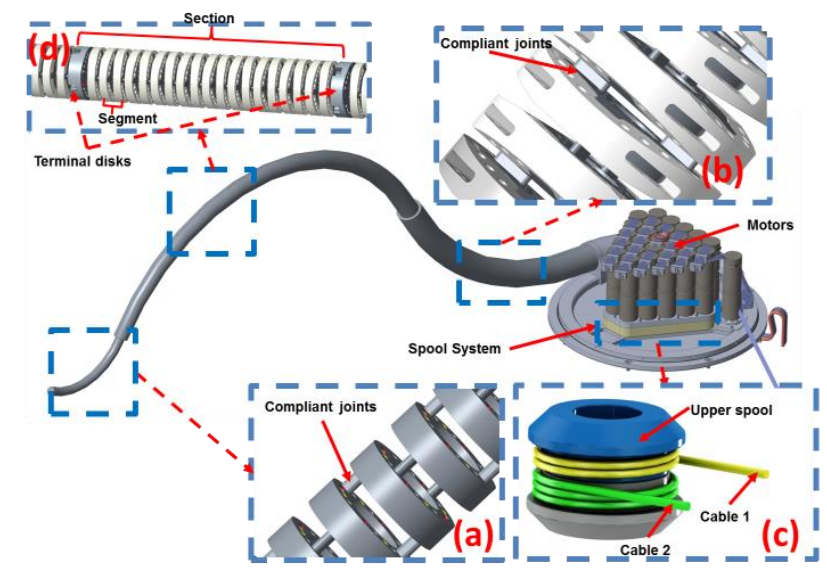

Figure 1. Generic representation of the main constitutive elements of the slender continuum robotic system: (a) rod based design of twin-compliant joint at the tip sections; (b) lamella based design of twin-compliant joint at the base sections (c) spool system (d) segment and section structure ${ }^{1}$;

Furthermore, to enable compact feeding-in of the robot, the design concept accommodated a coiling motion of continuum structure around a drum (which nests all the mechatronics of the system) using a geared actuation (see detail Figure 2(d)).

Bearing in mind that the continuum robot needs not only to be portable but also to have the capability to be attached to other (mobile) robots, it was decided that all the electronics (e.g. FPGAs, speed controllers for all motors, etc.) needed to be included in the actuation pack.

Thus, it could be noted that the concept of the slender continuum robot adopted here, while endeavouring to respond to tight engineering challenges, ends-up with very demanding mechatronics problems to be studied and addressed.

\section{KINEMATICS AND STATIC MODELS}

In this section, kinematic, static and compliant joint buckling models are presented to support the design and control of the continuum robot and the determination of its actuation specifications.

\section{A. Kinematics modelling}

In order to precisely control a multi-section continuum robot to reach a desired position, two maps of kinematics are needed in general. The first map refers to the relationship between task workspace (i.e. tool centre point - TCP - position) and the configuration space (i.e. orientation) of each independent articulated section of the continuum robot [13, 14]. The second one is between the configuration workspace and the joint/actuation space, which computes the actuation displacements (e.g. lengths of cable, flexible rods or pneumatic actuations) for each section to reach the desired configurations and vice versa [15-19].

\footnotetext{
${ }^{1}$ The length between two adjacent disks is termed as a segment; the length between two terminal disks is classified as a section; the terminal disks are where the actuation cables are attached (Figure 1(d)).
} 
Regarding the first map, a tip following approach for the navigation was developed and tested on one of our testing systems $[5,20]$. In particular, this strategy allows the user to navigate the tip through a restrictive environment, while the body of the arm manipulated to follow the path with minimal drift of approximately $15 \mathrm{~mm}$ in real-time.

Regarding the second map, the forward and inverse kinematics of twin-compliant joint structure were developed for controlling each individual section to the desired configurations, which has been validated on a testing system [21]. In this system, the continuum arm was structured with a tapered shape along the overall length, rendering the cables run through a tapered path, which requires a discussion of the kinematic model.

Table I

Nomenclature

\begin{tabular}{cc}
\hline \hline$l_{j}$ & length of the compliant joints, mm \\
\hline$l_{d}$ & thickness of the disks, mm \\
\hline $\mathrm{n}$ & number of segments in single section (see Figure 1(d)) \\
\hline
\end{tabular}

$l_{i}^{\prime}, l_{i}^{\prime \prime} \quad$ lengths of cable $i(i=1,2,3,4)$ in gaps 1 and 2 of segment $j(j=1,2,3 . . n)$ in a straight path, respectively, mm

${ }^{T} l_{i j}^{\prime},{ }^{T} l_{i j}^{\prime \prime} \quad$ lengths of cable $i(i=1,2,3,4)$ in gaps 1 and 2 of segment $j(j=1,2,3 . . n)$ in a tapered path, respectively, mm

\begin{tabular}{cc}
\hline$L_{i},{ }^{T} L_{i}$ & total length of cable $i(i=1,2)$ in straight and tapered path, respectively, mm \\
\hline$\beta_{i}$ & bending angle of joint $i(i=1,2)$, deg. \\
\hline $\mathrm{r}$ & pitch circle radius of the cable guide holes in section tip disk, $\mathrm{mm}$ \\
\hline$\Delta r$ & increment of $\mathrm{r}$ between adjacent disks, $\mathrm{mm}$ \\
\hline$\varphi$ & section tapered angle, deg. \\
\hline $\mathrm{S}$ & single section length, $\mathrm{mm}$ \\
\hline$\left(X_{p}, Y_{p}, Z_{p}\right)$ & the tip position of single section, $\mathrm{mm}$ \\
\hline$\theta$ & angle between $B_{1} B_{3}$ and axis $Y^{\prime}$, deg. \\
\hline \hline
\end{tabular}

The kinematic analysis is exemplified for the first segment of a single section. A schematic to support the understanding of the inverse kinematics model is presented in Figure 2. The bending and direction angles of single segment can be written in terms of given TCP position of single section $\left(\mathrm{X}_{\mathrm{p}}, \mathrm{Y}_{\mathrm{p}}, \mathrm{Z}_{\mathrm{p}}\right)$ and $\mathrm{n}$ [21]. By using the iteration method introduced in [21], the joint bending angles $\beta_{1}$ and $\beta_{2}$ can be found in order to reach the desired position for the section tip.

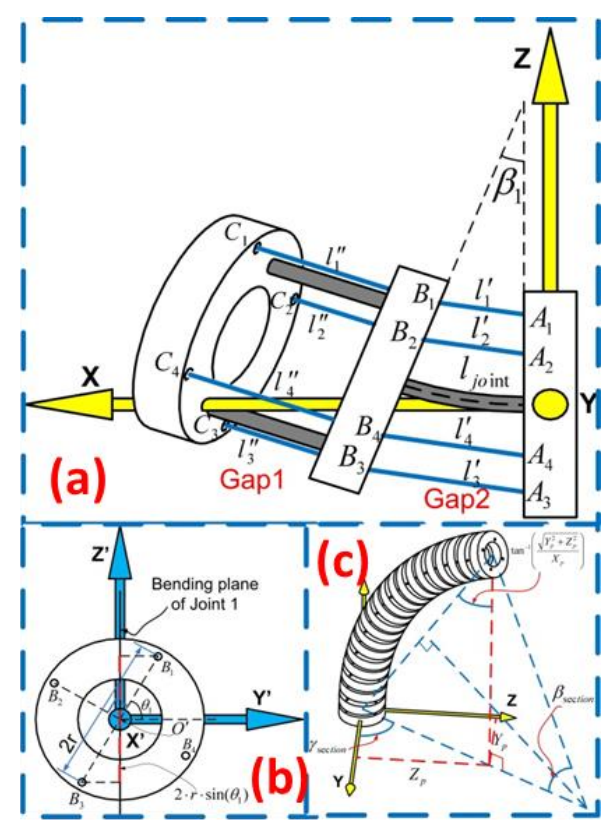

Figure 2. (a) Joint 1 bending section view (b) top view of disk B (c) section general view; 
Firstly, cable length kinematics of straight design (constant diameter) of the continuum robot is introduced, which is utilized in the following analysis for defining the kinematics of tapered design (as presented in the current paper). The cable lengths in gap 2 in straight path can be found by using (1):

$$
\left\{\begin{array}{l}
l_{2 i-1}^{\prime}=2\left(\eta_{1} \pm r \sin (\theta)\right) \lambda_{1} \\
l_{2 i}^{\prime}=2\left(\eta_{1} \pm r \cos (\theta)\right) \lambda_{1}
\end{array},(i=1,2)\right.
$$

Where $\eta_{1}=l_{j} / \beta_{1}$ and $\lambda_{1}=\tan \left(\beta_{1} / 2\right)$.

Similarly, the cables lengths in gap 1 can be obtained:

$$
\left\{\begin{array}{l}
l_{2 i-1}^{\prime \prime}=2\left(\eta_{2} \pm r \cos (\theta)\right) \lambda_{2} \\
l_{2 i}^{\prime \prime}=2\left(\eta_{2} \pm r \sin (\theta)\right) \lambda_{2}
\end{array}\right.
$$

Where $\eta_{2}=l_{j} / \beta_{2}$ and $\lambda_{2}=\tan \left(\beta_{2} / 2\right)$. Therefore, the overall section cable lengths can be found:

$$
L_{i}=n l_{i}^{\prime}+n l_{i}^{\prime \prime}+2 n l_{j},(i=1,2,3,4)
$$

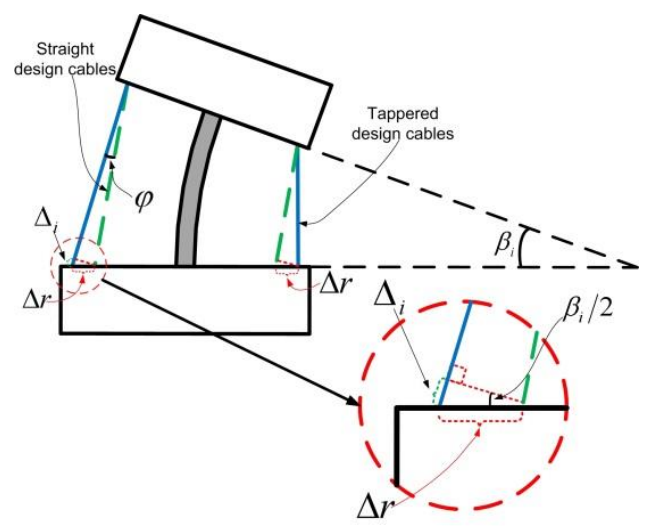

Figure 3. Cable kinematics model of tapered design

Since a tapered design is adopted for the current continuum robot, the cable lengths on their tapered paths are analysed based on the previous approach. The tapered angle is small $\left(\leq 0.5^{\circ}\right)$, hence, the cable length difference $\Delta_{i}$ between straight and tapered designs in a single segment can be expressed as:

$$
\left\{\begin{array}{l}
\Delta_{1}=\Delta r \sin \left(\beta_{1} / 2\right) \\
\Delta_{2}=\Delta r \sin \left(\beta_{2} / 2\right)
\end{array}\right.
$$

Hence, in the first segment of a single section, the cable lengths of gap 2 in tapered paths can be obtained by (5):

$$
\left\{\begin{array}{l}
{ }^{T} l_{(2 i-1) 1}^{\prime}=2\left(\eta_{1} \pm r \sin \left(\theta_{1}\right)\right) \lambda_{1} \pm \Delta_{1} \\
{ }^{T} l_{(2 i) !}^{\prime}=2\left(\eta_{1} \pm r \cos \left(\theta_{1}\right)\right) \lambda_{1} \pm \Delta_{1}
\end{array},(i=1,2)\right.
$$

And the cable lengths in gap 1 can be described as:

$$
\left\{\begin{array}{l}
{ }^{T} l_{(2 i-1) 1}^{\prime \prime}=2\left(\eta_{2} \pm r \cos \left(\theta_{1}\right)\right) \lambda_{2} \pm \Delta_{2} \\
{ }^{T} l_{(2 i) 1}^{\prime \prime}=2\left(\eta_{2} \pm r \sin \left(\theta_{1}\right)\right) \lambda_{2} \pm \Delta_{2}
\end{array},(i=1,2)\right.
$$

Since, the circle radius of the cable guide holes, $r$, increases from the tip to the base along the continuum robot, the cable lengths of segment $\mathrm{n}$ in gap 2 can be expressed:

$$
\left\{\begin{array}{l}
{ }^{T} l_{(2 i-1) n}^{\prime}=2\left(\eta_{1} \pm(r+\Delta r(2 n-2)) \sin \left(\theta_{1}\right)\right) \lambda_{1} \pm \Delta_{1} \\
{ }^{T} l_{(2 i) n}^{\prime}=2\left(\eta_{1} \pm(r+\Delta r(2 n-2)) \cos \left(\theta_{1}\right)\right) \lambda_{1} \pm \Delta_{1}
\end{array}\right.
$$


Similarly, the cable lengths of segment $\mathrm{n}$ in gap 1 can be written as:

$$
\left\{\begin{array}{l}
{ }^{T} l_{(2 i-1) n}^{\prime \prime}=2\left(\eta_{2} \pm(r+\Delta r(2 n-1)) \cos \left(\theta_{1}\right)\right) \lambda_{2} \pm \Delta_{2} \quad(i=1,2) \\
{ }^{r} l_{(2 i) n}^{\prime \prime}=2\left(\eta_{2} \pm(r+\Delta r(2 n-1)) \sin \left(\theta_{1}\right)\right) \lambda_{2} \pm \Delta_{2}
\end{array}\right.
$$

Hence, the overall cable lengths in tapered path can be calculated:

$$
\left\{\begin{aligned}
&{ }^{T} L_{(2 i-1)}= 2 n\left(\eta_{1} \pm(r+\Delta r(n-1) / 2) \sin \left(\theta_{1}\right)\right) \lambda_{1} \pm n \Delta_{1} \\
&+2 n\left(\eta_{2} \pm(r+\Delta r n / 2) \cos \left(\theta_{1}\right)\right) \lambda_{2} \pm n \Delta_{2}+n l_{j} \\
&= n\left(l_{(2 i-1)(n / 2)}^{\prime}+l_{(2 i-1)(n / 2)}^{\prime \prime}+l_{j}\right) \\
&{ }^{T} L_{(2 i)}= 2 n\left(\eta_{1} \pm(r+\Delta r(n-1) / 2) \cos \left(\theta_{1}\right)\right) \lambda_{1} \pm n \Delta_{1} \\
&+ 2 n\left(\eta_{2} \pm(r+\Delta r n / 2) \sin \left(\theta_{1}\right)\right) \lambda_{2} \pm n \Delta_{2}+n l_{j} \\
&= n\left(l_{(2 i)(n / 2)}^{\prime}+l_{(2 i)(n / 2)}^{\prime \prime}+l_{j}\right) \\
&(i=1,2)
\end{aligned}\right.
$$

Where $l_{(2 i-1)(n / 2)}^{\prime}+l_{(2 i-1)(n / 2)}^{\prime \prime}+l_{j}(\mathrm{i}=1$ and 2$)$ are the lengths of cable $1 \& 3$ in the gap number $\mathrm{n} / 2 ; l_{(2 i)(n / 2)}^{\prime}+l_{(2 i)(n / 2)}^{\prime \prime}+l_{j}(\mathrm{i}=1$ and 2) are the lengths of cable $1 \& 3$ in the gap number $n / 2$.

Therefore, it can be found that the overall cable lengths are equal to $n$ times of those of gap number $n / 2$. And the kinematics has been used in the real control algorithm of the tapered continuum arm.

\section{B. Actuation Force Estimation}

The specification for the actuators of the continuum robot has been done based on the estimation of the needed bending force for the compliant joints of the system; this has been done in the same manner as above, i.e. from a single gap/joint to multiple gaps/joints. Based on this estimation, the designer can select the motor and the actuation cables of the system while further enabling the design of the compliant joint (to avoid buckling).

\footnotetext{
Table II
}

Nomenclature used in this section

\begin{tabular}{cc}
\hline \hline$G_{L}$ & end load acting on a continuum robot section $(\mathrm{N})$ \\
\hline$G_{r}$ & weight of a continuum robot section $(\mathrm{N})$ \\
\hline$S^{\prime}$ & length of a segment $(\mathrm{mm})$ \\
\hline$S^{\prime \prime}$ & length of a joint (mm) \\
\hline$\beta_{s}^{\prime}$ & bending angle of single segment, deg. \\
\hline$\beta_{s}$ & bending angle of single section, deg. \\
\hline$F_{a}^{i}$ & active actuation force on cable 1 in gap $i(\mathrm{~N})$ \\
\hline$F_{t}$ & cable tension force $(\mathrm{N})$ \\
\hline$k$ & stiffness of twin compliant joints $(\mathrm{mm} / \mathrm{N})$ \\
\hline$F_{b}$ & force acting on the previous gap's backbone \\
\hline$A_{b}$ & moment arm of $F_{b}$ with respect to point $\mathrm{O}(\mathrm{mm})$ \\
\hline$F_{r}$ & component of $F_{b a s e}$ in the radial direction \\
\hline$A_{r}$ & the moment arm of $F_{r}$ with respect to point $\mathrm{O}$ \\
\hline \hline
\end{tabular}

As shown in Figure 4(a), two pairs of cables are attached on disk 1 in this model; one pair is in the horizontal plane and the other one is in the vertical plane.

Considering that the max actuation force is acting on cable 1, when the joint bends in vertical plane (Figure 4(b)), the moment equation of this configuration (with respect to point $\mathrm{O}$ ) can be expressed as:

$$
\begin{aligned}
& F_{a}^{1} r \cos \left(\beta_{1} / 2\right)+F_{t} r \cos \left(\beta_{1} / 2\right) \\
= & G_{L} r \sin \left(\beta_{1}\right)+G_{r} r \sin \left(\beta_{1} / 2\right)+k \beta_{1}+F_{t} r \cos \left(\beta_{1} / 2\right)
\end{aligned}
$$


Where $F_{a}^{1} r \cos \left(\beta_{s} / 2\right)$ is the moment generated by active actuation forces needed to bend the joint; $k \beta_{s}$ is the moment utilised to bend flexible backbone; $G_{L} r \sin \left(\beta_{s}\right)+G_{r} r \sin \left(\beta_{s} / 2\right)$ is the moment generated by the gravity of the whole unit and end load; $F_{t} r \cos \left(\beta_{s} / 2\right)$ is the moment caused by cable tension.

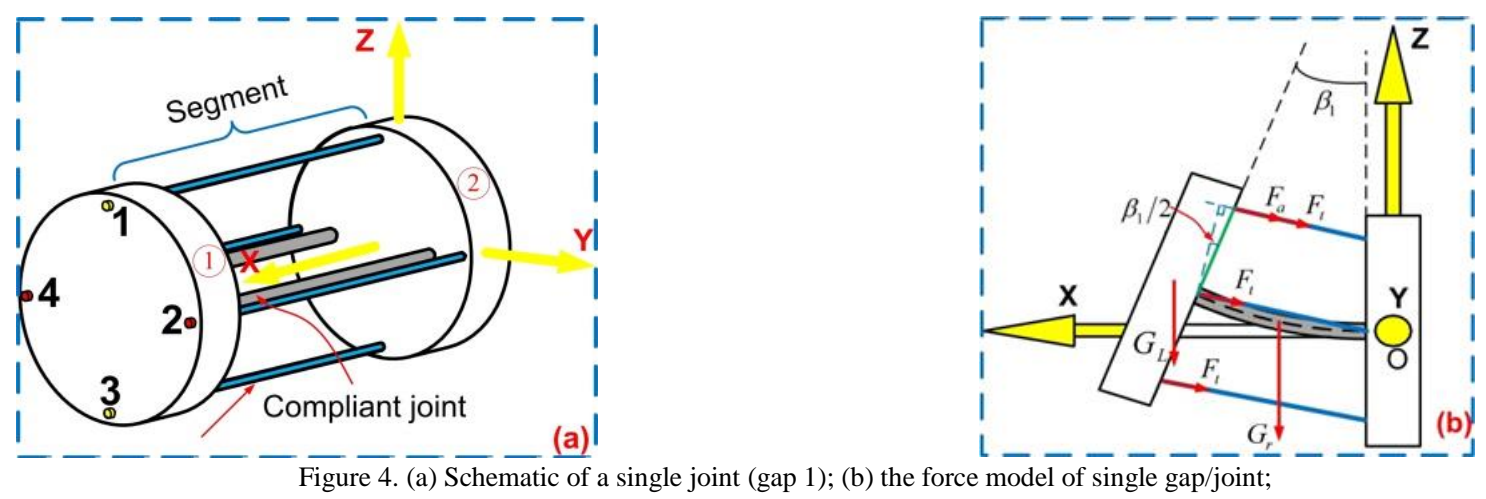

Since identical tension forces are applied on all the cables, the moments generated by tension forces can be omitted from both sides of (10). Hence, the actuation force for a single gap can be calculated as:

$$
F_{a}^{1}=\frac{\left(G_{L} \sin \left(\beta_{1}\right)+G_{r} \sin \left(\beta_{1} / 2\right)\right) r+k \beta_{1}}{r \cos \left(\beta_{1} / 2\right)}
$$

Further, the force analysis of multiple gaps/joints is presented, as shown in Figure 5. Regarding the first gap of the multiplejoint model, the force model is as the same as that of a single gap (see the previous case). Since the max bending force is applied on cable 1 when the robot bending in the vertical plane, the analysis is studied in this scenario (the other three cables are applied with tension force).

In respect of gap 2, there are two independent forces acting on it for forming its shape: (1) the load on the backbone $F_{b}$; (2) the interaction from the cables in radial direction $F_{r}$ (including four cables), as shown in Figure 5(a).

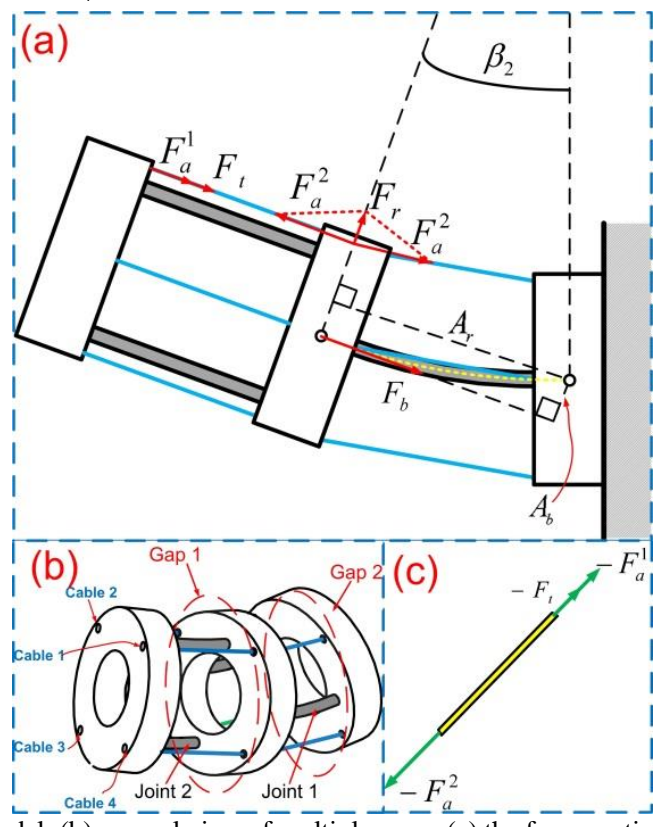

Figure 5. (a) Schematic of segment force model; (b) general view of multiple-gaps; (c) the forces acting on the length of cable 1 between disk 1 and 2;

Firstly, the load acting on the backbone is studied. Since four cables (two pairs) are applying force in the axial direction on disk 1 , which is parallel with the cables between disk 1 and $2, F_{b}$ can be expressed as:

$$
F_{b}=F_{a}^{1}+4 F_{t}
$$


Hence, the moment generated by $F_{b}$ applying on disk 2 with respect to point $\mathrm{O}$ can be obtained as:

$$
\begin{aligned}
M_{b} & =F_{b} \cdot A_{b} \\
& =2 \cdot\left(F_{a}^{1}+4 F_{t}\right) \cdot \frac{S}{\beta_{1}} \cdot \sin ^{2}\left(\frac{\beta_{1}}{2}\right)
\end{aligned}
$$

Where $A_{b}=2 \cdot \frac{S}{\beta_{1}} \cdot \sin ^{2}\left(\frac{\beta_{1}}{2}\right)$.

Secondly, the force in radial direction $F_{r}{ }^{1}$ of cable 1 is analysed. Since a cable needs to be pulled from its two ends to tension it, three forces act on the part of cable 1 between disk 1 and 2 as shown in Figure 5 (c), which can be described as:

$$
F_{a}^{2}+F_{a}^{1}+F_{t}=0
$$

Thus

$$
F_{a}^{2}=-F_{a}-F_{t}
$$

Due to the fact that cable 1 pulls disk 2 from both two directions with the same amount force $\left|F_{a}^{2}\right|$, the resultant force in the radial direction $F_{r}^{1}$, can be obtained as:

$$
\begin{aligned}
F_{r}^{1} & =F_{a}^{2} \cdot \sin \left(\beta_{1} / 2\right) \\
& =\left(-F_{a}^{1}-F_{t}\right) \cdot \sin \left(\beta_{1} / 2\right)
\end{aligned}
$$

Hence, the moment generated by $F_{r}{ }^{1}$ can be written as:

$$
M_{\text {cable_ } 1}=F_{r}^{1} \cdot A_{r}
$$

Where $A_{r}=S / \beta_{1} \cdot \sin \left(\beta_{1}\right)$.

Likewise, the moments generated by the other cables (cable 2, 3 and 4) can be obtained. Therefore, the total moment acting on disk 2 with respect to point $\mathrm{O}$, which is caused by the cables, can be expressed as:

$$
\begin{aligned}
M_{\text {cable }} & =-\left(F_{a}^{1}+4 F_{t}\right) \cdot \sin \left(\frac{\beta_{1}}{2}\right) \cdot \frac{S}{\beta_{1}} \cdot \sin \left(\beta_{1}\right) \\
& =-2\left(F_{a}^{1}+4 F_{t}\right) \cdot \frac{S}{\beta_{1}} \cdot \sin ^{2}\left(\frac{\beta_{1}}{2}\right) \cdot \cos \left(\frac{\beta_{1}}{2}\right)
\end{aligned}
$$

Since the max bending of a single section is $90^{\circ}$ and there are ten segments in each section, the max bending angle of each joint is $9^{\circ}$. Hence, it can be concluded that $\beta_{1} / 2 \leq 4.5^{\circ}$ and $\cos \left(\beta_{1} / 2\right) \geq 0.9969$, which can be omitted from the right side of (18):

$$
M_{\text {cable }}=-2\left(F_{a}^{1}+4 F_{t}\right) \cdot \frac{S}{\beta_{1}} \cdot \sin ^{2}\left(\frac{\beta_{1}}{2}\right)
$$

It is very clear that $M_{\text {cable }}$ and $M_{\text {backone }}$ are equal in magnitude and opposite in direction, which are counteracted with each other completely and the resultant moment is zero. Therefore, $F_{r}$ and $F_{b}$, which are the force from the previous joint, do not affect the calculation of the actuation force in the following joint. Regarding a single cable, the same magnitude of actuation force is applied on it along the full length of this cable.

According to (11), the actuation force for a segment can be expressed as:

$$
F_{a}=\frac{\left(G_{L} \sin \left(\beta_{s}^{\prime}\right)+G_{r} \sin \left(\frac{\beta_{s}^{\prime}}{2}\right)\right) \cdot \frac{S^{\prime}}{\beta_{s}^{\prime}} \cdot+k \beta_{s}^{\prime}}{r}
$$


And that of a section with $n$ segments:

$$
F_{a}=\frac{\left(G_{L} \sin \left(\beta_{s}\right)+G_{r} \sin \left(\frac{\beta_{s}}{2}\right)\right) \cdot \frac{S}{\beta_{s}} \cdot+k \beta_{s}}{r}
$$

Therefore, the auction force of an arbitrary section in a multiple-section continuum robot with a random bending shape can be calculated, which helps the designer determine the specifications of the actuation employed in the physical demonstrators.

\section{Compliant joint bucking modelling}

Buckling of the compliant joints is one of the major considerations when designing continuum robots based on this solution as this ensures the stability of the shape and behaviour of the system [22]. In this respect, Euler buckling theory is employed for evaluating the critical load above which the system loses structural stability:

$$
P_{c r}=\frac{\pi^{2} E I}{(K L)^{2}}
$$

Where $\mathrm{E}=$ Young's modules; $\mathrm{I}=$ area moment of inertia; $\mathrm{L}=$ unsupported length of column; $\mathrm{K}=$ column effective length factor, which value depends on the conditions of end support of the column: for both ends hinged (free to rotate), $\mathrm{K}=1.0$; for both ends fixed, $\mathrm{K}=0.50$; for one end fixed and the other end hinged, $\mathrm{K}=0.699$; for one end fixed and the other end free to move laterally, $\mathrm{K}=2.0$. In the case of this design of continuum arm one end of the compliant joint is fixed and the other end free to move laterally, hence, $\mathrm{K}=2$. Thus, based on (22) and considering the dimensions of the compliant joints (see design details in section IV) at each section of continuum robot, the critical load for bulking was found to be of a much higher value than the actual force caused by the wire tension. Additionally, FEA simulations (ANSYS) of the buckling load were carried out, resulting in a max. error $1.5 \%$ from the analytical solutions concluding that the dimensioning of the compliant joints is performed robustly.

\section{MECHATRONICS SYSTEM DESIGN AND DEVELOPMENT}

With the design concept (section II) and the key modelling aspects (section III) commented, in the following, the main aspects of the design and development of the continuum robot are commented.

\section{A. Continuum arm design}

The slender continuum arm has a total length of $1270 \mathrm{~mm}$, a tapered diameter, from $40 \mathrm{~mm}$ at its base to $13 \mathrm{~mm}$ at its tip (Figure 6 ), which renders an average diameter/length ratio of 0.023. Each of the twelve articulated sections (24 DoFs) was designed to be able to bend \pm 90 degrees.

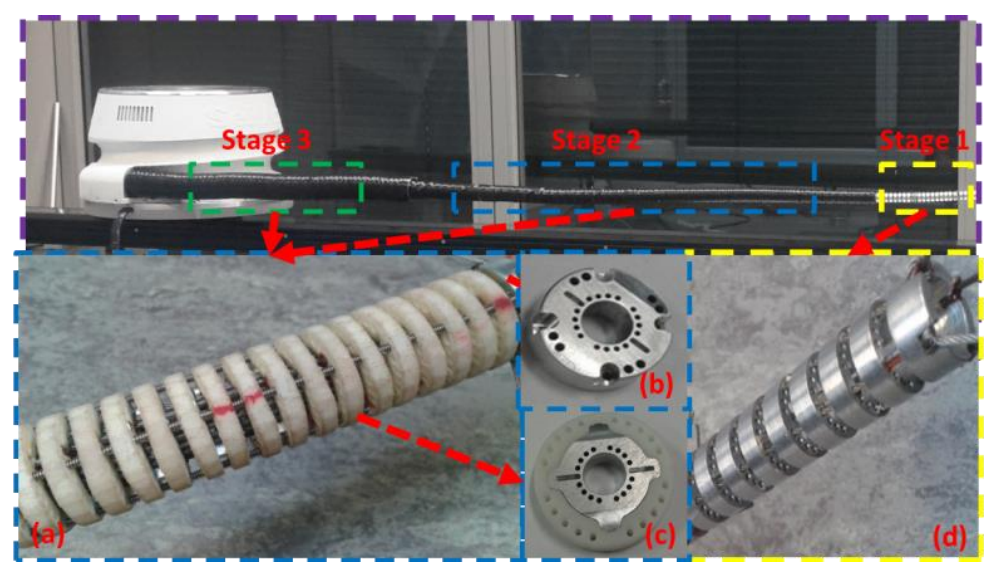

Figure 6. Key design elements of the slender continuum robot (a) structure of stage $2 \& 3$; (b) tip disk of each section in stage 2 and 3; (c) dual-material disks utilised in stage $2 \& 3$; (d) structure of stage 1;

The continuum arm consists of three stages of different dimensions:

(i) Stage 1 (Tip Sections (see Figure 6(d))) that consists of three sections of 13mm diameter (each with 2 DoF) that totals $150 \mathrm{~mm}$ in length; it is made of aluminium disks and NiTi compliant joints as rods (1.25mm diameter and $2.5 \mathrm{~mm}$ length). Stage 1 is intended to be used as active manipulator of the end-effector (e.g. cameras, machining heads) to perform the required repair task within a given confined working environment. 
(ii) Stage 2 (Middle Sections (see Figure 6(a))) consists of six sections of which three sections of $102 \mathrm{~mm}$ length and 20mm diameter and other three sections of $122 \mathrm{~mm}$ length and $22 \mathrm{~mm}$ diameter. Compliant joints in form of lamellae of $1.5 \mathrm{~mm}$ length and $1 \mathrm{~mm}$ thickness and from 2 to $4 \mathrm{~mm}$ width have been used. For Stage 2, a smart design solution has been adopted for each disks of continuum robot (except tip disk of each section (Figure 6(b))) that are made of Aluminium internal disk, to withstand the compressive forces along the longitudinal axis, and a lightweight Nylon outer layer to guide the steel cables, as shown in Figure 6(c); note that only radial forces act on the Nylon outer layer by the actuating cable when the continuum robot bends. This dual-material design can significantly save the weight of the continuum robot, allowing smaller motors to be utilised, which helps to minimize the weight and size of the actuation system; additionally, the inertia of the moving part of the robot will be minimised with positive implications on the dynamics of the system.

(iii) Stage 3 (Base sections (see Figure 6(a))) consists of three sections of constant diameter of $40 \mathrm{~mm}$ totalling a length of $678 \mathrm{~mm}$, and is constructed, for the same reasons, using the same dual-material design concept as the Middle Section. Compliant joints in form of plates of $1.5 \mathrm{~mm}$ length and $1 \mathrm{~mm}$ and $4.5 \mathrm{~mm}$ cross section have been used.

\section{B. Actuation system design}

The actuation pack indicted (Figure 7(a)) consists of the following main elements: (1) base plate - the rotational mechanism that allows coiling of the continuum robot around the drum, i.e. external cover; (2) motor pack assembly and cable guide to the continuum arm, (3) electrical and electronic command systems and mounting structure and (4) outer casing with a guide feature on the external surface to assist the continuum robot for its coiling motion. Thus, by rotating the base plate (1) relative to assembly of (2), (3) and (4), the continuum robot can be coiled and uncoiled on the outer casing, as shown in Figure 7 (a) that enables its feeding motion when the navigation is required.

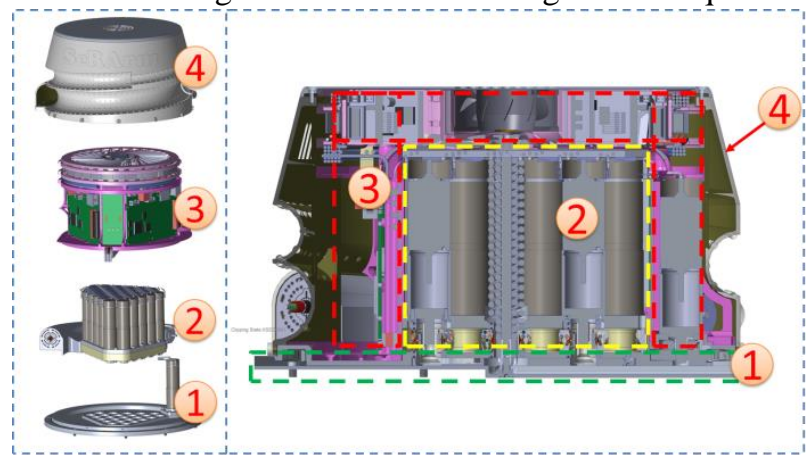

(a)

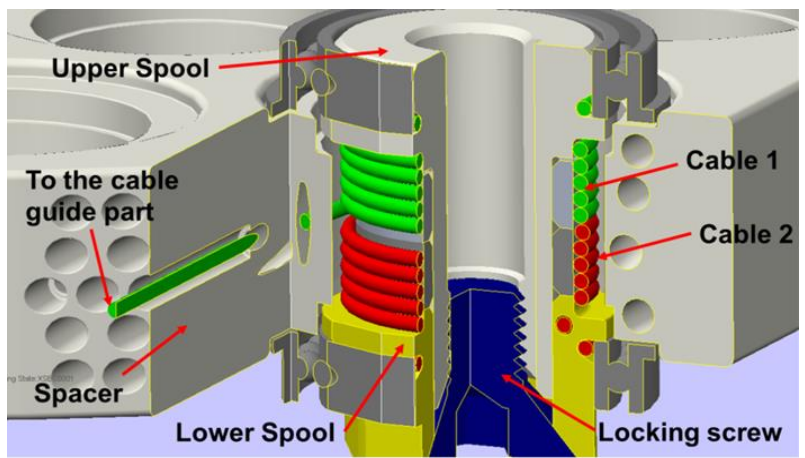

(b)

Figure 7. Design of actuation system (a) exploded view of sub assembles \& sectional view (b) section view of the spool system;

The rotation mechanism, consisting of an internal slew gearing ((1) - Figure 7(a)) was designed to have as low height as possible, whilst being able to provide sufficient drive so that it can advance the continuum robot. Thus, a motor mounted on the inner bearing is utilised to drive the outer bearing for rotating the casing. The gearhead motor (gear ratio: 236:1) has reas onably low backlash $\left(<0.8^{\circ}\right.$ ) and this enables the feeding motion of the continuum robot relatively quickly (approx. $0.002 \mathrm{~m} / \mathrm{s}$ ) in relation to a small number of motor encoder steps ( 1 motor encoder step $=0.5 \mu \mathrm{m}$ linear advancement of the robot).

As the continuum robot is designed based on four-cable concept, each motor is attached to an individual spool for actuating a pair of control cables. The pairs of cables serially run through the cable guide part, as indicated in (2)-Figure 7(a), and the body of the continuum robot up to a point where they separately attach to the tip of each individual section. The spool system, one of the key enablers of this design - Figure 7(b), has four main parts: the upper spool, the lower spool, the locking screw and the spacer. The upper spool attaches to the motor and the lower spool can make a concentric rotation relative to the upper one. Hence, the two cables, which are individually mounted on the upper and lower spools, allow mechanical adjustment of the tension in the cables by rotating the upper/lower spool. Further, the cables are helically coiled on the spool at the same diameter. Therefore, by rotating the spool by an angle, the same length of cables can be simultaneously released and pulled in from either the upper or lower spool, which ensures the continuum robot to keep a constant cable tension when bending. In this way, a constant proportional relationship between actuator motion and manipulator motion can be achieved. Each spool can provide a maximum stroke of $+/-200 \mathrm{~mm}$, so it allows each section bend $90^{\circ}$ and the continuum robot to coil on the outer casing.

\section{Architecture of the control system}

The low level control (LLC) of the continuum robot is based on two real-time embedded targets (sbRIO 9626) from National Instruments [3] both running as slaves to a host operating on a computer connected over Ethernet as presented in Figure 8. The boards each include 100 digital lines, 16 analog inputs, 4 analog outputs, and a Field-Programmable Gate Array (FPGA) Xilinx Spartan-6 LX45 capable of supporting the basic position control loops required to run each motor simultaneously. This configuration allows the control of 26 motors ( 24 for cable actuation, 1 for rotating the casing, 1 for machining spindle) and digital/analogue signals from the sensors (e.g. temperature control) in parallel. Each motor / encoder pair is connected via an 
electronic board containing a STMicroelectronics VNH5050A-E motor driver and a Texas Instruments MC3486D differential line receiver. Two boards are required as more digital lines are necessary than offered individually.

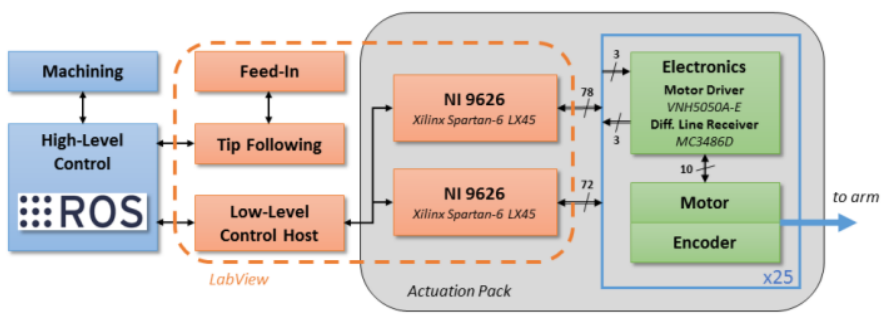

Figure.8. Control architecture of the continuum robot

The computer performs the user interaction and the conversion between configuration space of the continuum robot and the actuation motion (pull/release of the cables). Through the conversion, the code ensures that, for a required pose of some sections (e.g. tip and middle), the lower section (e.g. base) can changes its pose and thus, manipulate the entire upper structure keeping the subsequent configuration constant; this is of particular importance when the continuum robot reached a specific position and some sections need to keep their pose constant to avoid possible collisions within the working environments.

The high level control (HLC) has been implemented in ROS [4] and communicates to the LLC through TCP/IP with the aid of ROSbridge protocols [5]. The HLC is able to generate paths for the tip following using environmental data, or create successive linear trajectories based on the current heading established by a camera embedded in the tip. Further, the task planner is employed to determine what actions are required to achieve the goal, such as deciding what point the continuum robot needs to be navigated to that allows machining of the target point.

\section{MODES OF CONTROL FOR THE CONTINUUM ROBOT}

\section{A. Tip-following command}

The tip-following, presented in [15], allows the robot to navigate through a restrictive environment by organising a succession of poses as the system is advanced. This means the tip can be piloted through the scenario, either by user interaction or planned paths, while the body is manipulated such that it follows the route taken. Unlike some alternate methods, this algorithm does not require the environmental data or sensor feedback as it minimises the errors from its own path.

For every small step the distal end (i.e. tip) makes, the configuration is updated by an optimisation routine and an equivalent step at the base. This is built on an objective function that provides a scalar performance factor created from the Euclidean error at the tip and the drift from the desired path. To characterise the drift from the desired path, the Cartesian points of the path are compared to the points that simulate the shape of the continuum arm. Each step of the path is measured against the closest part of the robot. The maximum value is then taken as the current drift. For example, solutions from this algorithm can be generated in $0.410 \mathrm{~s}$ on average, meaning pre-planned paths circa 500 steps can be completed in less than 5 minutes while maintaining the poses of the continuum robot under $15 \mathrm{~mm}$ of drift from the path and $\pm 0.75 \mathrm{~mm}$ error at the tip on average.

As the flowchart in Figure 10 shows, the algorithm needs three inputs: the trajectory to follow, the current configuration and the previous trajectory to the current point. The configuration and prior path is combined with the new trajectory if provided. For each step of the trajectory, the process is looped. The coordinates are added to the desired path and the base position is advanced equidistant to the spacing of the tip points. Using the optimization changes to the arrangement are found that minimize the errors, then the pose is updated, and the process is repeated. The configuration poses created are provided at the end in a single array, it is also possible to output poses to a First-In First Out to actuate the poses on-the-fly.

Trajectories can be invoked using three possible methods: a path can be provided that is determined by the shape of a desired final configuration, or the HLC can plan a path through a CAD representation of the environment, or, the user can adjust the heading of the tip section by using images captured by a camera, then command a straight movement from that position of the desired length in what is referred to as "Endoscope Mode". 

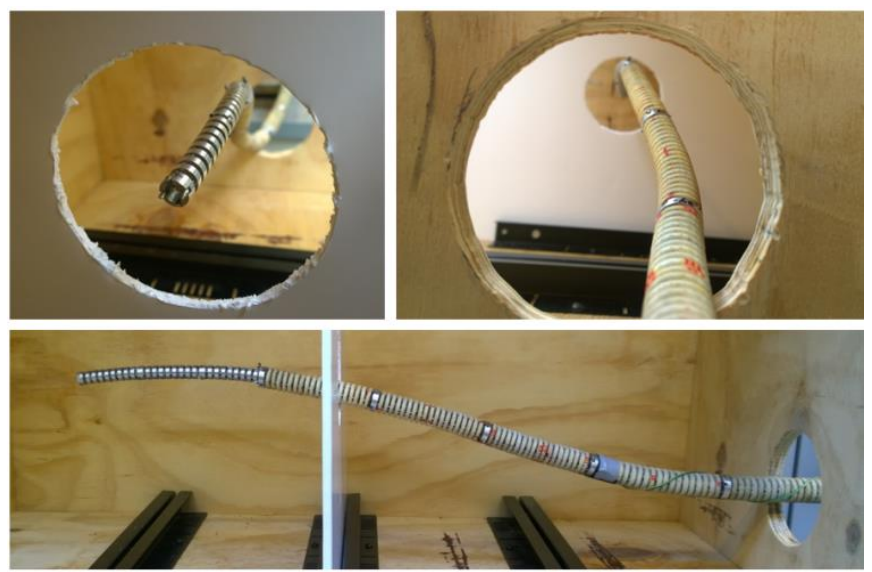

Figure 9. Tip following through a simple demonstration environment

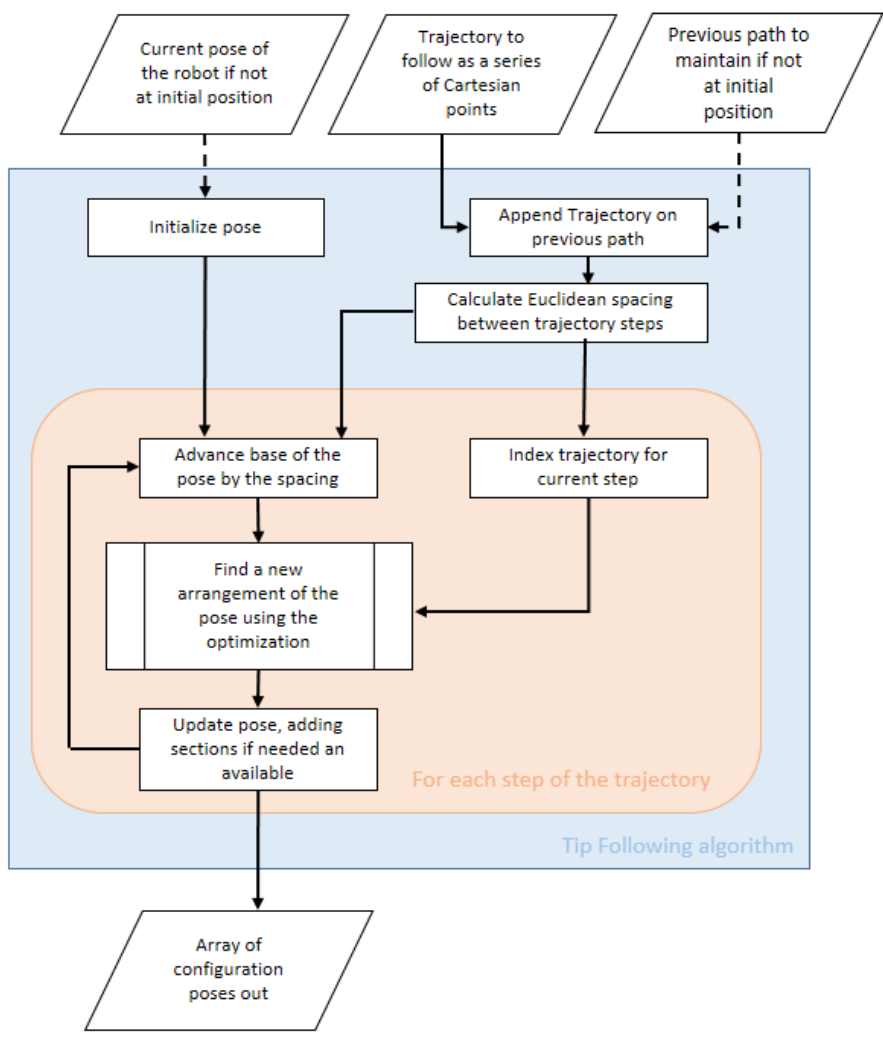

Figure 10. Flowchart of the Tip Following algorithm

Using the final configuration method, the continuum robot was navigated through a simple box to demonstrate the functionality of the algorithm. Figure 9 depicts the prototype in-situ.

\section{B. Feeding-in/out command}

Since the previously described tip-following movement needs the continuum robot to be advanced from the base, a mechanism is required to perform this action. Traditional approaches in the field use a linear track, industrial robot arm, or a mobile robot platform. The challenges with these solutions are that they require space for at least the length to be advanced to be provided by the external platform (e.g. linear stage); not all scenario environments have enough external freedom to allow this. As such, the feed-in system chosen for this robot is an actuated rotating drum to which the arm can be actively coiled around. Figure 11 shows the system in both states (i.e. coiled and uncoiled). 


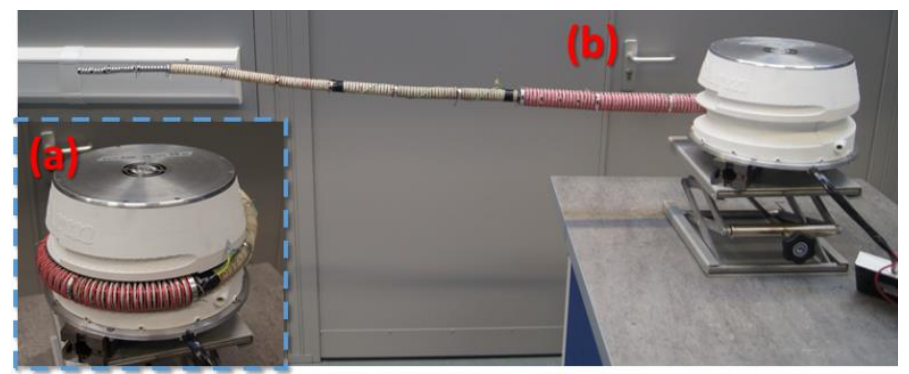

Figure 11: The continuum robot in its A) coiled state and B) uncoiled

Due to the behaviour of the joints, coiling the system creates lateral errors, while ascending the helix creates a vertical shift. Therefore, a corrective movement is required to ensure the arm is advanced along the trajectory expected by the tip-following movement; in this case horizontal from the base connection. The simplest action to take is to create an "S-bend" with the next two sections. This can fix the vertical and horizontal drift and certify the route is parallel to the target, see Figure 12.
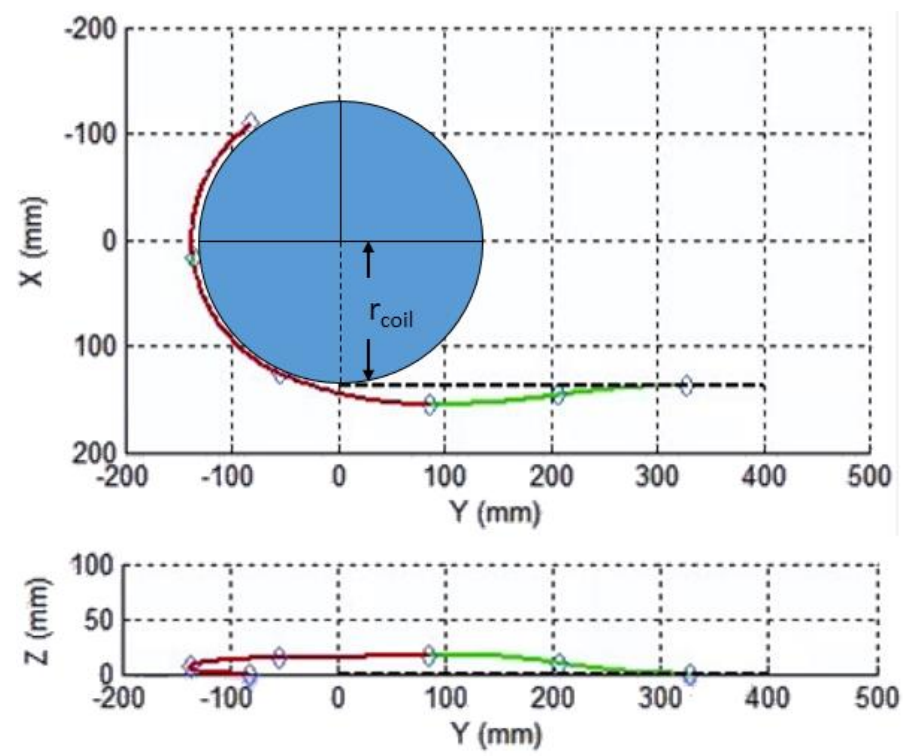

Figure 12 Graphical depiction of uncoiling process. Sections are separated by blue diamonds; red sections are being coiled, while green sections are correcting the errors a) Top view b) Side view.

Table III

Nomenclature in this section

\begin{tabular}{cc}
\hline \hline$\Delta x, \Delta y$ & horizontal and vertical error in coiling (mm) \\
\hline$P_{t i p}$ & Cartesian point at distal end of the coil (mm) \\
\hline$r_{\text {coil }}$ & radius of the coil (mm) \\
\hline$R_{t i p}$ & rotation matrix to the distal end \\
\hline$\omega_{x}, \omega_{z}$ & horizontal and vertical angular error rad. \\
\hline \hline$t_{k}$ & time instant, (s) \\
\hline$q\left(t_{k}\right)$ & joint variables at the time instant \\
\hline$J^{-1}\left(q\left(t_{k}\right)\right)$ & inverse Jacobian at time instant \\
\hline$v_{d}\left(t_{k}\right)$ & instantaneous velocities at time instant \\
\hline \hline
\end{tabular}

The required S-bend is calculated by another optimization routine. In this case the objective function is constructed of four parts to represent the errors. The target is a line parallel to the $\mathrm{Y}$-axis, in this case, at $\mathrm{x}=\mathrm{r}_{\text {coil }}$ and $\mathrm{z}=0$. The drift therefore can be found as: 


$$
\begin{aligned}
& \Delta x=\left|P_{\text {tip }, x}-r_{\text {coil }}\right| \\
& \Delta z=\left|P_{\text {tip }, z}-0\right|
\end{aligned}
$$

where $\mathrm{P}_{\text {tip }}$ is the co-ordinate of the tip. Since the rotation matrix at the tip is known, the pitch and yaw angles to the trajectory are determined by

$$
\begin{aligned}
& P^{\prime}=R_{t i p} \times\left[\begin{array}{l}
0 \\
0 \\
1
\end{array}\right] \\
& \omega_{x}=\left|\arctan \left(P_{x}^{\prime} / P_{y}^{\prime}\right)\right| \\
& \omega_{z}=\left|\arctan \left(P_{z}^{\prime} / P_{y}^{\prime}\right)\right|
\end{aligned}
$$

Combined, the objective function can be written as

$$
f=\Delta x+\Delta z+100 \omega_{x}+100 \omega_{z}
$$

where the angles, calculated in radians, are weighted by a factor of 100. In this way the performance values are balanced with a slight bias towards the orientation, since these errors have the greater effect on the quality of the Tip Following

\section{Machining command}

Once the continuum robot navigates to the required position, only the last three sections (6 DoF) are used for moving an endeffector so that it can perform an active task, e.g. machining. A numerical implementation based on the inverse of the Jacobian [23] was implemented for a previous instant in time, such that:

$$
q\left(t_{k+1}\right)=q\left(t_{k}\right)+J^{-1}\left(q\left(t_{k}\right)\right) v_{e}\left(t_{k}\right) \Delta t
$$

where ${ }^{t_{k} \in \mathfrak{R}^{+}}$is the time instant, ${ }_{k+1} \in \mathfrak{R}^{+}$is the next period of time, $J^{-1}\left(q\left(t_{k}\right)\right) \in \mathfrak{R}^{6 \times 6}$ is the inverse of the Jacobian for the last 6 DoF in the time instant, ${ }_{e}\left(t_{k}\right) \in \mathfrak{R}^{6 \times 1}$ is the vector of instant velocities of the end-effector, and $\Delta t \in \mathfrak{R}$ is an scalar that minimize the error.

This method implies that the joint variables, $q$, that correspond to a given desired pose of the end-effector are precisely computed only when the error between the desired end-effector pose and the candidate end-effector pose is reduced to within a given threshold; therefore, the computing time depends on the dynamic characteristics that are produced by the error of the differential equations. The inverse kinematic (26) can be used to produce linear and arc/spline trajectories for the end-effector to respond to the needs of active tasks to be done by the continuum robot.

\section{PERFORMANCE CHARACTERIZATION}

The validation of the capabilities of this complex mechatronic system has been performed through a succession of tests to check some key characteristics to targeted demonstrations on mock-up industrial environments (e.g. aero-engines).

\section{A. Payload test}

The inherent flexibility of the continuum arm could lead to compliant safe interactions with the surrounding environment. However, the arm is supposed to have reasonable payload capabilities to enable manipulation of the end-effectors for performing active tasks. Hence, a range of payload tests was undertaken to evaluate the end load carrying capability of the system. 


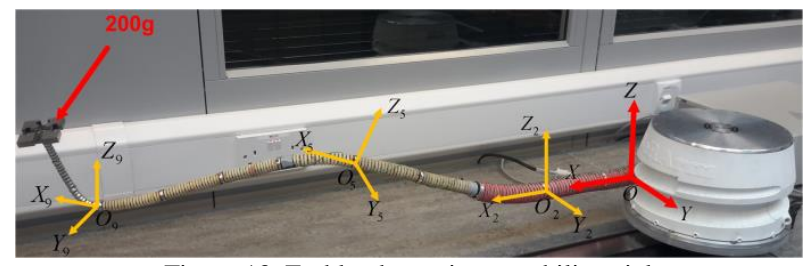

Figure 13. End load carrying capability trial

Different end loads were mounted on the top of the arm in full-uncoiled position while taking some critical poses that replicate some configurations resembling those needed to the industrial application (i.e. repair of gas turbine engines). For example, as shown in fig 13Figure, the continuum arm was set to the following configuration:

$$
\left[\begin{array}{l}
\beta_{2} \\
\gamma_{2} \\
\beta_{5} \\
\gamma_{5} \\
\beta_{9} \\
\gamma_{9}
\end{array}\right]=\left[\begin{array}{c}
30^{\circ} \\
90^{\circ} \\
45^{\circ} \\
210^{\circ} \\
70^{\circ} \\
90^{\circ}
\end{array}\right]
$$

The deflections seem to be linearly dependent on the end-load (Table IV); although the deflections seem high in absolute values they might be regarded relatively small when compared to the full length of the continuum robot $(1256 \mathrm{~mm})$.

Table IV

Deflections of the full length arm versus end loads

\begin{tabular}{cc}
\hline \hline End load Mass $(\mathrm{g})$ & TCP deflection $(\mathrm{mm})$ \\
\hline \hline 50 & 5.4 \\
\hline 100 & 10.3 \\
\hline 150 & 15.7 \\
\hline 200 & 21.1 \\
\hline \hline
\end{tabular}

Not considering any active positioning systems, there could be two simple ways to minimise the deflection of the arm caused by end-effector. One is to control the tip position via a camera embedded at the tip of the arm by the operator and another one by employing a light-weight end effector (in current design $45 \mathrm{~g}$ with deflection of $4.8 \mathrm{~mm}$ ). For the reported continuum robot both methods have been employed which allowed making compensations of these errors.

\section{B. Navigation test in engine model}

The evaluation of the continuum robot evolved towards the testing of the technology in mock-up industrial environments, i.e. inspection and machining. Thus, in order to perform the inspection task the continuum robot needs to be navigated to reach the first stage of Intermediate Pressure Compressor (IPC). According to the construction of a gas turbine engine (Rolls-Royce Trent XWB), an example of navigation path is generically described (Figure 14(a), (b) \& (c))):

- $19^{\circ}$ bend in $+\mathrm{Z}$ direction in the plane $\mathrm{XOZ}$ (arc length: $132 \mathrm{~mm}$ )

- Straight path $(280 \mathrm{~mm})$ with a $19^{\circ}$ tilted angle

- $25^{\circ}$ bend in -Y direction in the plane XOY (the arc length: $349 \mathrm{~mm}$ );

- Straight path $(300 \mathrm{~mm})$ with a $19^{\circ}$ tilted angle;

- $25^{\circ}$ bend in $+Y$ direction in the plane XOY (the arc length: $100 \mathrm{~mm}$ ). 


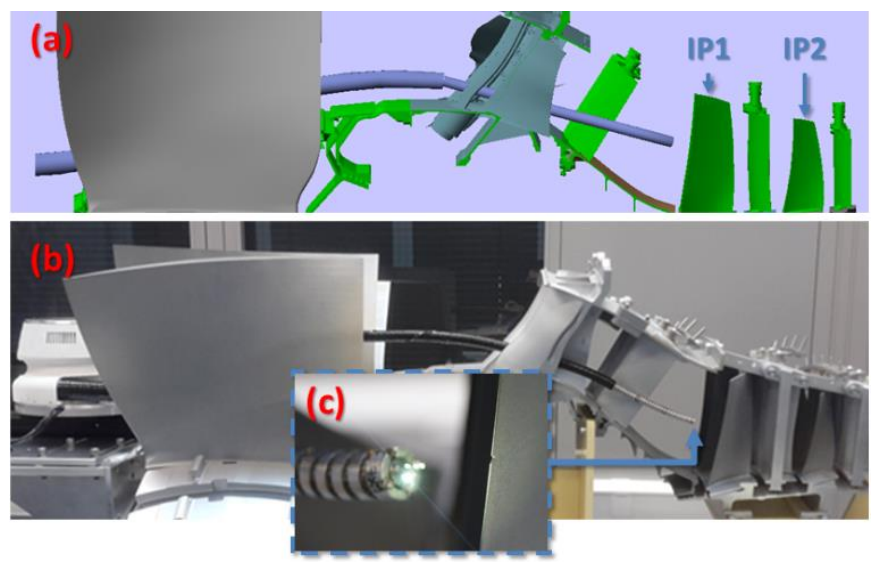

Figure 14: Views of graphical representation of the navigation of the continuum robot into the gas turbine engine (a) and trial on an engine mock-up (RollsRoyce XWB) with detail of the observed mechanical defect on the aerofoil (c).

With the continuum robot successfully navigating from the front of the engine at a max. $10 \mathrm{~mm}$ deviation from the path, the cameras were utilised to observe a mechanical defect of an aerofoil; this trial has been performed repeatedly proving the robustness in achieving this task.

\section{Inspection and machining trail}

Using the endoscopic mode, the tip of the continuum robot was navigated through the stators to the $1^{\text {st }}$ and $2^{\text {nd }}$ stages of the engine to reach a mechanical defect (i.e. notch) on a leading edge of a blade and perform a blending (arc shape stress relief) on it. This task is done by using the miniature end-effector that (see Fig. 15) equipped with two cameras and a machining spindle achieving high spindle speeds (i.e. 10,000 rpm).

Once determining the planar magnitudes (max dimensions) of the defect on the aerofoil, the operator decides on the profile required to be machined (as arcs) so that the part complies with quality regulatory standards for stress relief on the blade. By smoothing these stress raising features on the critical components, it is possible to lengthen the operational time of the engine without taking it off the wing (and disassemble) and thus to significantly reduce costs and lead times.

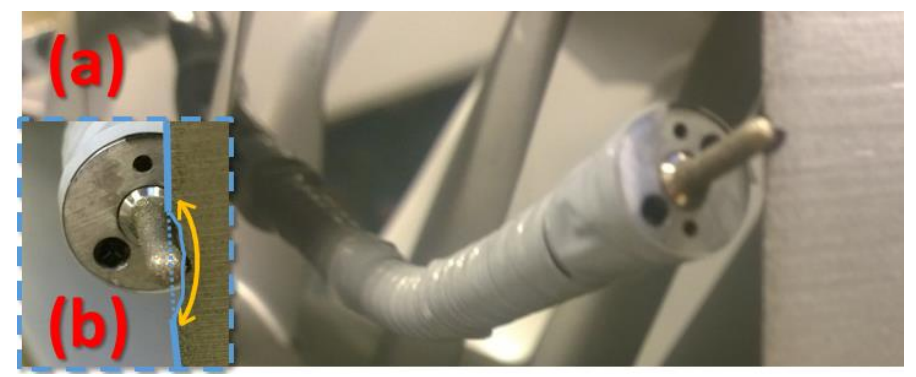

Figure 15: View of the continuum robot within the mock-up aero engine when machining (blending) a virtual defect on an aerofoil;

The inverse kinematic (26) can be used to produce linear and arc trajectories for the end effector such as is shown in Figure 15. Arcs have been performed in this case and the scallop feature on the mock-up blade has been performed at a length accuracy of $0.2 \mathrm{~mm}$. This was considered satisfactory for this application.

\section{CONCLUSIONS}

The paper reports on a succession of full scientific and engineering route to materialise a novel concept of a slender continuum robot to be utilised on a very demanding application, i.e. in-situ inspection and machining for repair of aeroengine parts without the need of its disassembly. The concept relies on a tapered arm of 12 sections based on compliant joints that is able to coil around the main body of the continuum robot, which contains the actuation back and electronics, making it a truly portable system. Kinematic models have been developed to enable the control of the multi-section robot reaching the desired position while the force model supports the selection of actuation system and dimensioning of the compliant joints to avoid their buckling. The realisation of the 24 DoFs continuum robot uses compliant joints of various stiffness along its length and allows $\pm 90^{\circ}$ bend angles for each section while the 2 pairs of actuation cables drove the demand of developing an original spooling system to allow smooth and compensatory lengths of two opposite cables for each actuated section. Particular attention was given to make a compact mechatronic system where all 24 motors actuating the cables and 1 motor for coiling the continuum robot as well as control boards and speed controllers have been carefully included in the same actuation pack. The control 
architecture has been presented on which the main two critical algorithms, i.e. tip following for allowing the continuum robot navigation into intricate workspaces and the machining mode using the last $6 \mathrm{DoF}$ of the system; these algorithms have been tested in laboratory setups with satisfactory results. Further, a set of performance characterisation (accuracy, repeatability, reproducibility) tests have demonstrated that the robot can take poses at a level of precision that allows its use for in-situ repair tasks. Finally, the slender continuum robot has been successfully tested by navigating between aerofoils into mock-up aeroengine, access the $2^{\text {nd }}$ stage of compressor and perform inspection and machining paths to replicating motions needed to repair of a blade.

The paper draws to the attention of robotic community an interesting and challenging application of continuum robots for in-situ repair of space constrained that drives the need to redefine design concepts, models and mechatronics of such systems.

\section{ACKNOWLEDGMENT}

The research leading to these results has received funding from the European Union Seventh Framework Programme (FP72011) under grant agreement no. 284959 (MiRoR: http://www.miror.eu/) and Rolls-Royce Plc.

\section{REFERENCES}

[1] RICHARD WOLF BLENDING SCOPE. AVAILABLE: HTTP://WWW.BLENDING-SCOPE.COM/

[2] OLYMPUS VIDEOSCOPES. AVAILABLE: HTTP://WWW.OLYMPUS-IMS.COM/EN/REMOTE-VISUAL-INSPECTION/VIDEOSCOPE/

[3] MACHIDA BLENDING BORESCOPE. AVAILABLE: HTTP://WWW.MACHIDASCOPE.COM/BLENDINGSCOPES

[4] SNAKE-ARM ROBOTS FOR AIRCRAFT ASSEMBLY. AVAILABLE: SOLUTIONS/AEROSPACE/AEROSPACE-CASE-STUDY/

[5] X. Dong, M. RafFles, S. C. Guzman, D. AXinte, AND J. Kell, "Design and analysis of a family of SNaKe aRm Robots conNected by COMPLIANT JOINTS," MECHANISM AND MACHINE THEORY, VOL. 77, PP. 73-91, 2014.

[6] N. SimaAn, R. TAylor, and P. Flint, "A Dexterous system For laryngeal Surgery," in Robotics and Automation, 2004. PRoceEdings. ICRA'04. 2004 IEEE INTERNATIONAL CONFERENCE ON, 2004, PP. 351-357.

[7] J. Ding, K. Xu, R. Goldman, P. Allen, D. Fowler, And N. SimaAn, "Design, Simulation and eValuation of Kinematic alternatives for INSERTABLE ROBOTIC EFFECTORS PLATFORMS IN SINGLE PORT ACCESS SURGERY," IN ROBOTICS AND AUTOMATION (ICRA), 2010 IEEE INTERNATIONAL CONFERENCE ON, 2010, PP. 1053-1058.

[8] FESTO. (2011). BIONIC TRIPOD 3.0. AVAILABLE: HTTP://WWW.FESTO.COM/CMS/EN_CORP/11371.HTM

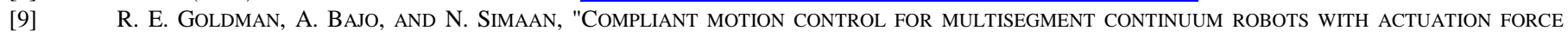
SENSING," ROBOTICS, IEEE TRANSACTIONS ON, VOL. 30, PP. 890-902, 2014.

[10] C. Li and C. D. Rahn, "Design of Continuous backbone, CABle-Driven Robots," Journal of MECHANICAL DESIGN, vol. 124, PP. 265-271, 2002 .

[11] X. Dong, M. Raffles, S. Cobos-Guzman, D. Axinte, and J. Kell, "A Novel Continuum Robot Using Twin-Pivot Compliant Joints: DESIGN, MODELING, AND VALIDATION," JOURNAL OF MECHANISMS AND ROBOTICS, VOL. 8, P. 021010, 2016.

[12] FESTO. (2010). BIONIC HANDLING ASSISTANT-FLEXIBLE AND COMPLIANT MOVEMENT. AVAILABLE: HTTP://WWW.FESTO.COM/CMS/EN_CORP/9655_10219.HTM

[13] G. S. CHIRIKJIAN, "THEORY AND APPLICATIONS OF HYPER-REDUNDANT ROBOTIC MANIPULATORS," CALIFORNIA INSTITUTE OF TECHNOLOGY, 1992.

[14] G. S. CHIRIKJIAN AND J. W. BURDICK, "A MODAL APPROACH TO HYPER-REDUNDANT MANIPULATOR KINEMATICS," ROBOTICS AND AUTOMATION, IEEE TRANSACTIONS ON, VOL. 10, PP. 343-354, 1994.

[15] I. A. GRAVAGNE AND I. D. WALKER, "KinEMATIC TRANSFORMATIONS FOR REMOTELY-ACTUATED PLANAR CONTINUUM ROBOTS," IN ROBOTICS AND AUTOMATION, 2000. PROCEEDINGS. ICRA'OO. IEEE INTERNATIONAL CONFERENCE ON, 2000, PP. 19-26.

[16] I. A. GRAVAGNE AND I. D. WALKER, "ON THE KINEMATICS OF REMOTELY-ACTUATED CONTINUUM ROBOTS," IN ROBOTICS AND AUTOMATION, 2000. PROCEEDINGS. ICRA'OO. IEEE INTERNATIONAL CONFERENCE ON, 2000, PP. 2544-2550.

[17] M. W. HANNAN AND I. D. WALKER, "ANALYSIS AND EXPERIMENTS WITH AN ELEPHANT'S TRUNK ROBOT," ADVANCED ROBOTICS, vOL. 15, PP. 847-858, 2001.

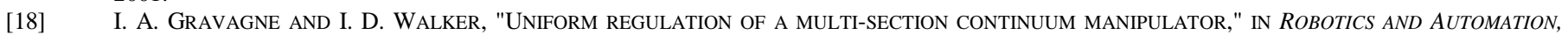
2002. PROCEEDINGS. ICRA'02. IEEE INTERNATIONAL CONFERENCE ON, 2002, PP. 1519-1524.

[19] K. XU AND N. SimAAN, "ANALYTIC FORMULATION FOR KINEMATICS, STATICS, AND SHAPE RESTORATION OF MULTIBACKBONE CONTINUUM ROBOTS VIA ELLIPTIC INTEGRALS," JOURNAL OF MECHANISMS AND ROBOTICS, VOL. 2, P. $011006,2010$.

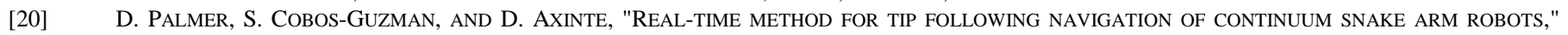
RoBotICS AND AUTONOMOUS SYSTEMS, VOL. 62, PP. 1478-1485, 2014.

[21] X. Dong, M. Raffles, S. C. Guzman, D. Axinte, and J. Kell, "A Novel Snake Arm Robot Using Twin-Pivot Compliant Joints: Design, MODELING AND VALIDATION," JOURNAL OF MECHANISMS AND ROBOTICS.

[22] N. LOBONTIU, COMPLIANT MECHANISMS: DESIGN OF FLEXURE HINGES: CRC PRESS, 2002.

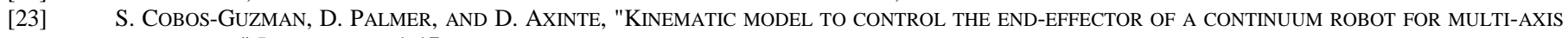
PROCESSING," ROBOTICA, PP. 1-17. 